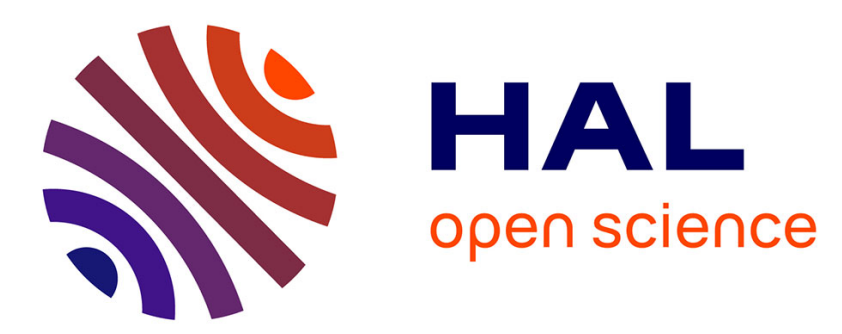

\title{
L'accueil des enfants : enjeux des réformes et appel aux entreprises dans quatre pays européens
}

\author{
Anne-Marie Daune-Richard, Marie-Thérèse Letablier
}

\section{To cite this version:}

Anne-Marie Daune-Richard, Marie-Thérèse Letablier. L'accueil des enfants: enjeux des réformes et appel aux entreprises dans quatre pays européens. Politiques sociales et familiales, 2011, 103, pp.33-46. halshs-00583015

\section{HAL Id: halshs-00583015 https://shs.hal.science/halshs-00583015}

Submitted on 11 Apr 2011

HAL is a multi-disciplinary open access archive for the deposit and dissemination of scientific research documents, whether they are published or not. The documents may come from teaching and research institutions in France or abroad, or from public or private research centers.
L'archive ouverte pluridisciplinaire HAL, est destinée au dépôt et à la diffusion de documents scientifiques de niveau recherche, publiés ou non, émanant des établissements d'enseignement et de recherche français ou étrangers, des laboratoires publics ou privés. 


\title{
L'accueil des enfants : enjeux des réformes et appel aux entreprises dans quatre pays européens
}

\author{
\begin{tabular}{c|l} 
& \\
\cline { 2 - 2 } Anne-Marie Daune-Richard & CNRS/Laboratoire d'économie et de sociologie du travail
\end{tabular} \\ - Université d'Aix-Marseille. \\ Marie-Thérèse Letablier CNRS/Centre d'économie de la Sorbonne-Université \\ Paris-1 Panthéon-Sorbonne.
}

Mots-clés : Comparaison européenne - Modes de garde Politique familiale.

\begin{abstract}
Afin de répondre à l'urgente nécessité d'augmenter l'offre d'accueil des enfants, les entreprises sont conviées à participer à cet objectif et à s'engager dans un soutien plus actif à l'égard de leurs salariés ayant des enfants. Cet article examine comment les entreprises sont mobilisées autour de la question de l'accueil des enfants dans quatre pays de I'Union européenne, I'Allemagne, la France, I'Italie et le Royaume-Uni. Au préalable, cet appel aux entreprises est situé dans le contexte plus large des réformes des politiques d'accueil, soulignant les convergences entre les objectifs assignés à ces réformes en lien avec les injonctions de I'Union européenne, et les diverses formes de mise en œuvre. Sont ainsi mises en évidence différentes logiques d'implication des entreprises, articulées à divers modèles d'intervention de l'État et de partenariats public-privé.
\end{abstract}

\begin{abstract}
u cours des dix dernières années, les politiques d'accueil des jeunes enfants ont été réformées dans la plupart des pays européens afin de mieux répondre aux besoins des parents et aux injonctions de I'Union européenne en faveur d'un développement accru des structures d'accueil, conformément aux objectifs fixés au Sommet de Barcelone en 2000. Cette injonction prend place dans un contexte général de limitation des dépenses publiques qui incite les gouvernements à rechercher des partenaires pour financer et piloter le développement de ce secteur d'activité. Les associations, et plus largement la société civile, ont été appelées à participer à ce développement, comme les entreprises et les partenaires sociaux. L'implication des entreprises revêt une importance et des formes variables selon le paysage institutionnel propre à chaque pays, en fonction des outils mis en place pour encourager cette implication et des modes de gouvernance
\end{abstract}

spécifiques à chaque contexte. L'objectif de cet article est de comparer comment les entreprises ont été associées ou impliquées dans les réformes de l'accueil des enfants dans quatre pays de I'Union européenne, I'Allemagne, la France, I'Italie et le Royaume-Uni, qui partaient de situations très différenciées à la fin des années 1990 lorsque la stratégie européenne s'est mise en place. L'étude s'est appuyée sur une recherche comparative fondée sur trois séminaires au cours desquels des chercheurs ayant réalisé des études sur ce sujet dans les quatre pays ont échangé, à partir d'une grille commune, leurs analyses des réformes des politiques d'accueil, de leurs conséquences sur la gouvernance des services d'accueil, et sur l'implication des entreprises dans ce secteur (1) (DauneRichard et Letablier, 2009). En premier lieu, on verra comment se situe cette politique d'activation des entreprises dans les enjeux des réformes de l'accueil des enfants par l'accent mis sur les priorités assignées à ces réformes dans chacun des pays. Puis seront observées les modalités d'appel aux entreprises, notamment du point de vue de la recomposition des relations entre public et privé dans le secteur de l'accueil des enfants.

\section{Les réformes de l'accueil des enfants et leurs enjeux}

L'appel réitéré de la Commission européenne en faveur d'une "modernisation des politiques familiales » afin de mieux les adapter aux nouveaux défis du $X X I^{\mathrm{e}}$ siècle a été diversement entendu. Plusieurs pays ont néanmoins engagé des réformes, plus ou moins substantielles, de leur politique familiale pour la rendre plus effective dans le soutien apporté aux parents qui travaillent, et leur

\footnotetext{
1) Cet article s'appuie sur une recherche financée par la Caisse nationale des Allocations familiales (CNAF) en 2008-2009 sur I'implication des entreprises dans l'accueil des enfants. Les auteures remercient les chercheurs ayant participé à ce travail : Sophie Rouault et Mechthild Veil pour l'Allemagne, Stefania Sabatinelli pour l'Italie, Colette Fagan et Mark Smith pour le Royaume-Uni, Sophie Odena et Francesca Petrella pour la France, ainsi que Danielle Boyer qui a suivi ce projet à la CNAF.
} 
permettre de mieux concilier travail et vie familiale. $\mathrm{Si}$, depuis plus d'une décennie, I'enjeu principal est de répondre aux besoins des parents qui travaillent et de permettre aux mères de rester dans l'emploi, d'autres enjeux se sont superposés, notamment ceux liés à l'évolution démographique, à la nécessité de resserrer la cohésion sociale et de lutter contre la pauvreté des enfants. Si la fixation d'objectifs chiffrés en matière d'accueil des enfants au Sommet de Barcelone en 2002 (2) a pu laisser craindre une certaine subordination de la politique de l'enfance à des objectifs économiques et d'emploi, les développements de la politique communautaire au cours des premières années de ce siècle indiquent une autre préoccupation liée à l'éducation et au bien-être des enfants, formulée en termes d'investissement social.

Enjeux au niveau communautaire : de la notion de charge à celle d'investissement social

Depuis une quinzaine d'années, les réformes des politiques sociales promues par l'Union européenne vont de pair avec un renouvellement de la conception de l' «État social» orienté vers un «État d'investissement social » (Giddens, 1998; Palier, 2005) visant à encourager l'investissement en capital humain et à soutenir l'emploi plutôt que le revenu. Cette "troisième voie », intermédiaire entre une voie purement néolibérale et une voie sociale-démocrate, visait à contenir les dépenses publiques dans un contexte de vieillissement démographique et de croissance ralentie. Elle marquait un tournant dans la conception des politiques sociales par une attention portée aux enfants en tant qu'ils concentrent l'espoir d'un retour sur investissement en termes d'éducation, de performances scolaires et de bien-être. En changeant le regard sur les enfants, passant d'une vision en termes de "charge » et de coût à une vision en terme "d'investissement social», la réorientation des politiques se veut mieux adaptée à la construction d'une société de la connaissance.

Le premier grand enjeu des réformes des politiques d'accueil est d'abord un défi quantitatif lié à la nécessité de développer l'offre d'accueil afin de répondre aux besoins des parents qui travaillent et d'augmenter la participation des mères au marché du travail, considérée comme une condition nécessaire à l'autonomie économique des femmes et à l'égalité avec les hommes. Le second cible la qualité des modes d'accueil et met l'accent sur la dimension éducative de l'accueil, s'appuyant sur des résultats de recherches qui montrent l'avantage pour une société de développer l'éducation précoce des enfants (3). L'idée est de recentrer l'intervention publique sur les besoins des enfants et pas seulement sur ceux des parents. Les débats entourant la mise en place $d^{\prime} u n$ droit opposable en France ou d'un droit individuel de l'enfant à un accueil éducatif, comme dans les pays nordiques, ou encore d'un droit à une éducation préscolaire pour les plus grands des jeunes enfants, confortent la notion de "droit des enfants » à un environnement qui permette leur développement cognitif et leur bienêtre. En effet, si au premier rang des arguments justifiant les réformes des politiques d'accueil figure la nécessité de soutenir les parents pour qu'ils participent au marché du travail (4), d'autres arguments mettent la question de la qualité de I'accueil au cœur du débat. Ces arguments sont développés par l'Organisation de coopération et de développement économiques (OCDE) dans son rapport "Starting Strong II », qui insiste sur l'importance de la dimension éducative de l'accueil des enfants, ainsi que sur l'importance de l'éducation précoce, non seulement au regard des performances des enfants en matière $d^{\prime}$ apprentissage, mais aussi au regard d'une certaine égalité des chances (OCDE, 2006). On assiste ainsi à un changement d'approche de la « garde » des enfants qui souligne I'importance du développement cognitif des enfants, ainsi que le fait qu'il ne peut être I'apanage des seuls parents. Ce recadrage marque un tournant radical dans I'approche de l'enfant dans des pays comme I'Allemagne où l'éducation des jeunes enfants était l'apanage des mères. Plusieurs pays ont ainsi institué un droit des enfants à une éducation préscolaire.

C'est dans ce contexte général que se retrouve posée la question de l'accueil des enfants, en tant que responsabilité de l'État, pour répondre à la fois aux besoins induits par l'incitation faite aux mères

(2) Au sommet de Barcelone en 2002, des conclusions et des objectifs explicites ont été définis en matière d'offre de services d'accueil. Tout en confirmant l'objectif du plein emploi, le Conseil européen de 2002 recommandait aux États membres de supprimer les éléments dissuasifs à la participation des femmes à l'emploi et, par conséquent, de développer les services d'accueil des enfants d'ici 2010 pour au moins $33 \%$ des enfants âgés de moins de 3 ans et $90 \%$ des enfants de 3 ans à l'âge de la scolarité obligatoire. L'importance de ces objectifs a été rappelée lors de l'adoption par le Conseil européen des lignes directrices pour l'emploi 2008-2010.

(3) Pour un aperçu de ces recherches, voir Letablier et al., 2009, chapitre 5

(4) Les États peuvent investir dans les services d'accueil des enfants pour diverses raisons. Une raison se réfère au fait que la disponibilité de services d'accueil a un impact sur le taux d'emploi des mères, et une participation importante des mères au marché du travail favorise une plus grande égalité entre les hommes et les femmes, contribuant à viabiliser les systèmes de sécurité sociale dans un contexte de vieillissement de la population. Une autre raison, liée à la précédente, concerne la contribution à l'augmentation de la fécondité, notamment par une réduction de la tension entre travail et responsabilités familiales. Toutefois, si les arguments en faveur du développement des modes d'accueil des enfants sont désormais bien connus, de nombreux États membres sont encore loin d'atteindre les objectifs de Barcelone, les barrières étant davantage financières qu'idéologiques car la plupart des États membres ont pris des initiatives afin d'augmenter l'offre de services. 
de se porter sur le marché du travail, à la nécessité urgente de réduire la pauvreté des enfants et d'augmenter leur niveau d'éducation dans une société orientée vers la connaissance. Investir dans les enfants est donc devenu I'un des mots d'ordre de la dernière décennie, non seulement pour assurer la pérennité de la protection sociale, mais aussi pour promouvoir l'égalité des chances face à l'éducation et à la formation et ainsi déjouer le déterminisme de l'héritage social. Ainsi, Gøsta EspingAndersen (2008) suggère de combiner une stratégie $d^{\prime}$ emploi des mères soutenue par une offre $d$ 'accueil abordable par des congés parentaux rémunérés, et par des transferts familiaux susceptibles d'élever le revenu des familles au-dessus du seuil de pauvreté. Les États membres ont diversement répondu à ces recommandations, non seulement en fonction de ce qui existait jusqu'alors mais aussi en fonction des moyens alloués à cette politique, des instruments utilisés et des acteurs impliqués. Les réformes des politiques d'accueil des enfants ont donc été plus ou moins substantielles, plus ou moins radicales, selon les situations de départ et les priorités des réformes (5).

\section{Des priorités diverses dans les quatre pays}

À la fin des années 1990, I'accueil des enfants d'âge préscolaire varie considérablement d'un pays à l'autre. Parmi les quatre pays considérés ici, seule la France peut s'enorgueillir de disposer d'une politique d'accueil des enfants, instituée, instrumentée et fonctionnelle, bien que les besoins soient loin d'être couverts. Depuis les années 1970, en effet, la politique familiale s'est progressivement réorientée vers un soutien aux parents qui travaillent en mettant en place une politique d'accueil des enfants articulée à un système de prestations. Mais cette politique a fini par aboutir à un empilement de mesures et de prestations la rendant opaque pour les parents. Le principe de l'intervention publique est néanmoins bien admis et celui d'une régulation publique bien établi. Dans les années 1980, l'école maternelle est progressivement généralisée à tous les enfants âgés de 3 ans à 6 ans, l'accent étant mis sur la dimension éducative avant la scolarité obligatoire ainsi que sur l'accès à tous les enfants quelles que soient leur origine sociale ou la situation de leurs parents, la gratuité étant le gage de l'égalité des chances pour les enfants. Cette dimension éducative s'est même étendue jusqu'au début des années 2000 à une partie des enfants âgés de 2 ans à 3 ans. De fait, la politique d'accueil des enfants renvoie, en France, essentiellement à l'accueil des enfants de moins de 3 ans ainsi qu'à l'accueil périscolaire. Dans ce contexte, la priorité est, au début de cette décennie, d'augmenter l'offre d'accueil pour les enfants âgés de moins de 3 ans afin de répondre aux besoins des parents qui travaillent de simplifier le système de prestations et de le rendre plus visible pour les parents, de réformer ce système pour le rendre plus incitatif au travail des mères.

Ainsi, plusieurs réformes ont eu lieu au cours de la dernière décennie visant à mieux répondre aux besoins des familles, notamment à la diversification des besoins, et à mobiliser de nouveaux acteurs. La loi réformant le système de prestations d'accueil des jeunes enfants en 2004 vise à simplifier le système de prestations tout en réaffirmant le principe d'action du gouvernement français : préserver et développer la liberté de choix des parents vis-àvis du mode d'accueil de leurs enfants. Elle vise également à rendre accessible à un plus grand nombre de parents l'accès à un mode d'accueil individuel par une assistante maternelle. Le statut des assistantes maternelles a ensuite été révisé afin de réguler cette profession, notamment par une contractualisation de la relation d'emploi. De fait, l'augmentation de l'offre d'accueil repose en grande partie sur le développement de l'accueil par les assistantes maternelles, même si les parents continuent de déclarer leur préférence pour un mode d'accueil dans une structure collective et si l'accueil collectif reste le mode d'accueil le plus accessible financièrement aux familles modestes (Voisin, 2009). Le plan Petite enfance 2007-2012 prévoit une augmentation substantielle du nombre de places d'accueil en même temps qu'une diversification de l'offre afin de mieux s'ajuster à la diversité des demandes. L'objectif est également d'encourager le développement de partenariats avec de nouveaux acteurs, et en premier lieu avec les entreprises.

Jusque dans les années 1990, dans les trois autres pays (Allemagne, Italie et Royaume-Uni), la garde des enfants est moins une affaire d'État qu'une affaire de famille, et surtout de mères. Ces pays peuvent, en effet, être qualifiés de " maternalistes » en raison de l'importance du rôle des mères dans les soins aux enfants, une importance reconnue - bien que sous des formes diverses - dans la législation et les principes de la protection sociale. Aucun des trois pays ne dispose de l'équivalent français de l'école maternelle, même si en Italie, la majorité des enfants de plus de 3 ans sont accueillis dans de

5) Il importe de rappeler ici que les quatre pays ne consacrent pas le même pourcentage de leur produit intérieur brut (PIB) à la politique familiale : tandis que I'Allemagne consacre 3,2\% de son PIB aux prestations sociales " familles-enfants », la France y consacre 2,5\%, le Royaume-Uni 1,7\% et I'Italie 1,1\% (Fagnani et al., 2008). Si on rapporte ces dépenses aux dépenses de protection sociale, la part de celles allouées à la fonction famille-enfants s'élève à 10,8\% en Allemagne, $8 \%$ en France, 6,2 \% au Royaume-Uni et 4,2\% en Italie. Ces résultats recouvrent cependant de fortes variations selon le type de prestations : ainsi, les prestations en nature sont évaluées à 0,8\% du PIB en Allemagne, 0,5\% en France et en Italie (mais n'incluent pas les écoles maternelles) et 0,4\% au Royaume-Uni (Fagnani et al., 2008). 
telles structures, celles-ci n'étant pas nécessairement de statut public. En Allemagne comme au Royaume-Uni, les mères se retirent du marché du travail après la naissance d'un enfant, pour reprendre ensuite une activité, généralement à temps partiel, afin de pouvoir combiner leur emploi avec les exigences de la maternité. Les mères allemandes sont éligibles pour une allocation parentale assimilable à un salaire maternel, car n'étant pas conditionnée par une activité professionnelle antérieure à la naissance. En dépit de différences entre les trois pays concernant les prestations de soutien à la garde des enfants, les enfants de moins de 3 ans sont principalement gardés par leur mère, les structures d'accueil étant rares.

Les réformes engagées à la fin des années 1990 au Royaume-Uni avec la mise en place du premier plan de développement des modes d'accueil des enfants ("National Childcare Strategy ») par le nouveau gouvernement travailliste en 1998 ont fait l'effet d'une petite révolution dans l'approche de la garde des jeunes enfants. La stratégie vise en premier lieu à lutter contre la pauvreté des enfants dont le niveau est très préoccupant (Unicef, 2000 et 2006). L'ambition est également d'augmenter le taux d'emploi des mères et de faciliter la conciliation entre travail et vie familiale. Le programme "Sure Start » mis en place en 1998-1999 s'adresse à tous les enfants et accorde un soutien renforcé aux enfants et aux familles les plus en difficulté par des actions ciblées sur certaines zones et régions. Afin de mieux articuler accueil et éducation, les services d'accueil de l'enfance ont été récemment confiés à l'unité "Sure Start » rattachée au ministère de l'Éducation et placée sous l'autorité conjointe des ministères de l'éducation et du travail. Il s'agit pour cette unité de mettre en œuvre le programme "Sure Start » destiné à offrir à chaque enfant le meilleur départ possible dans la vie, en réunissant les services d'accueil, d'éducation préscolaire, de santé et de soutien familial. Un deuxième plan mis en place en 2004 (Ten years strategy) conforte cette orientation et, en 2006, I'adoption du Childcare Act en définit le cadre légal. Les mesures prises depuis dix ans se résument dans la poursuite des quatre objectifs suivants: flexibilité, disponibilité, qualité et solvabilité.

Alors que la pauvreté des enfants et leur bien-être continuent d'occuper une place centrale dans les recherches internationales de I'Unicef, de I'OCDE (Whiteford et Adema, 2007) et d'Eurostat (2008), c'est plutôt l'accès à l'éducation précoce qui a constitué l'enjeu majeur des réformes en Allemagne après la diffusion des résultats des enquêtes PISA de l'OCDE qui ont ébranlé les convictions relatives à la supériorité de l'éducation maternelle sur toute autre (Fagnani, 2010). Parallèlement, depuis dix ans, I'Allemagne s'est engagée dans un processus de réformes réformes profondes de sa politique familiale afin de la moderniser, c'est-à-dire de mieux I'adapter aux évolutions sociétales, et notamment aux défis démographiques et à l'évolution du travail des mères (Fagnani, 2010). Deux réformes ont ainsi marqué un tournant décisif dans la réorientation de la politique familiale allemande: la loi entrée en vigueur au $1^{\text {er }}$ janvier 2005 sur l'extension de l'accueil de la petite enfance (loi TAG) et la réforme "phare» du congé parental, entrée en vigueur en janvier 2007. Pour la première fois, la loi TAG pose publiquement la question de la garde collective des enfants de moins de 3 ans comme étant un enjeu sociétal majeur. Cette loi se donne pour objectif de stopper la diminution de l'offre d'accueil en crèche dans les Länder de l'Est et d'en augmenter le nombre dans ceux de l'Ouest à I'horizon 2013, pour satisfaire les besoins évalués à $33 \%$ des enfants de ce groupe d'âge, soit 2,1 millions d'enfants, et ainsi répondre aux objectifs fixés au niveau communautaire. La loi TAG propose aussi de rééquilibrer l'offre d'accueil entre offre individuelle et offre collective, en faveur des structures collectives selon une répartition de deux tiers et un tiers. Aujourd'hui, 88 \% des enfants de moins de 3 ans qui ne sont pas gardés par l'un des parents le sont dans des structures collectives (Veil et Rouault, 2009).

En Italie, les réformes se sont effectuées en ordre dispersé, ce pays n'ayant pas de politique familiale véritablement instituée au niveau national. La garde des jeunes enfants reste principalement le fait des mères et/ou des grands-mères. Depuis la loi « crèches » de 1971, la politique d'accueil des jeunes enfants incombe aux communes qui peuvent recevoir un financement des régions pour couvrir les coûts de fonctionnement. En 1997, une nouvelle loi sur l'enfance prévoit des financements de trois ans alloués au développement de modes d'accueil, mais le renouvellement de ces financements a été interrompu. Dans les années 2000, les réformes se succèdent mais avec des objectifs parfois contradictoires. Un programme spécial de création de crèches (Piano Straordinario Nidi) est mis en place en 2006 par le gouvernement Prodi, suivi d'un accord entre le gouvernement, les régions et les collectivités locales pour créer un réseau intégré de crèches sur l'ensemble du territoire afin de " promouvoir le bien-être et le développement des enfants, soutenir le rôle éducatif des parents et faciliter la conciliation des temps du travail et de la famille ». Ce plan exceptionnel prévoit la création de soixante-cinq mille places d'accueil dont vingt mille dans des classes pour les enfants âgés de 2 ans à 3 ans. Les efforts doivent être concentrés sur les régions du sud de l'Italie afin d'assurer un taux de couverture de $6 \%$ minimum dans les régions les plus dépourvues. Aujourd'hui, le plan a survécu à la chute du gouvernement Prodi en 2008. 


\section{Des réformes du pilotage des politiques d'accueil}

Non seulement le contenu des politiques et leurs objectifs prioritaires ont été réformés mais leur mode de pilotage et de gouvernance ont aussi été repensés. Ces réformes s'inscrivent dans un contexte général d'évolution des réglementations, de diversification des solutions d'accueil, de raréfaction des ressources publiques et d'émergence de nouveaux acteurs dans ce champ politique. En Italie, les réformes dans un contexte politique de subsidiarité des responsabilités publiques ne semblent pas déboucher sur une harmonisation du pilotage de l'accueil.

Les évolutions ont été les plus marquées en Allemagne. Pendant longtemps, I'intervention des autorités publiques dans l'accueil des jeunes enfants a été subsidiaire par rapport à celle du tiers secteur, et c'est ce qui a changé depuis le début des années 2000, notamment sous l'effet de la réunification. L'objectif affiché d'augmenter les taux d'activité des femmes ainsi que le taux de natalité dont le niveau est particulièrement faible dans ce pays, a poussé l'État fédéral à s'engager dans la voie d'une politique familiale plus interventionniste mettant l'accent sur l'urgente nécessité de développer des structures d'accueil pour les enfants afin de faciliter la conciliation entre travail et vie familiale des parents (Klammer et Letablier, 2008 ; Fagnani et Math, 2007). La loi TAG a force d'obligation pour les Länder et les communes qui doivent assurer le financement des structures d'accueil, aidés par l'État fédéral à hauteur de 1,5 milliard d'euros par an. Depuis 2005, les Länder ont la possibilité de financer des services privés lucratifs et ne sont plus limités au financement des associations, parentales ou charitables. Ces réformes ont fait de l'accueil de la petite enfance un enjeu conflictuel du fédéralisme en Allemagne : alors que le Bund (symboliquement en la personne de la ministre fédérale à la famille) se présente désormais comme le fer de lance de la refonte des politiques familiales (et des mentalités), les Länder et les communes sont en grande partie chargés du financement des mesures annoncées à Berlin et de leur mise en œuvre. Cette répartition des prérogatives, conflictuelle dans un contexte de compression des dépenses publiques locales, fonde les appels au réalisme des collectivités locales. Selon Sophie Rouault et Mechthild Veil (Daune-Richard et Letablier, 2009), le niveau fédéral est donc celui où s'élabore le référentiel d'action publique : il s'agit moins de soutenir les familles nécessiteuses comme au Royaume-Uni que de soutenir les couples biactifs et de contribuer à la politique d'activation des mères. Le niveau fédéral se présente aussi comme celui où s'animent les réseaux et où se mettent en place les partenariats entre acteurs publics et privés nécessaires à la mise en œuvre de la stratégie fédérale. Dans cette structure politique fédérale, l'absence d'une branche " famille » dans le système de sécurité sociale, associée au fait que le développement des crèches n'était pas considéré comme une priorité dans les Länder de l'Ouest, a laissé l'initiative à la société civile, aux églises et aux entreprises pour développer l'offre d'accueil des jeunes enfants. Mais, depuis un jugement de la Cour constitutionnelle fédérale de 1967, le cadre constitutionnel s'est complexifié car ce jugement attribue la compétence en matière de fourniture de services d'accueil de la petite enfance aux communes et non plus aux organisations caritatives, particulièrement à celles d'origine confessionnelle qui fournissent toujours une part importante des services à l'enfance et à la jeunesse. Les communes ont donc la responsabilité d'évaluer les besoins et de planifier leur action en matière d'offre d'accueil. Elles peuvent toutefois déléguer une partie de ces prérogatives à des prestataires publics, privés ou caritatifs (Veil, 2010).

En France, le pilotage de la politique publique $d^{\prime}$ accueil s'inscrit dans un paysage institutionnel qui articule un partage des responsabilités entre les caisses d'Allocations familiales (CAF), les conseils généraux et les municipalités. Les deux premiers fixent le cadre réglementaire et financier tandis que les municipalités définissent et mettent en place les politiques en fonction des besoins de leur population et de leurs priorités, c'est-à-dire celles des élus locaux et des valeurs qu'ils cherchent à promouvoir. Depuis les années 1980, une politique contractuelle menée par les CAF est mise en place. Piliers de cette politique, les contrats crèches ont été transformés en 2006 en contrats enfance et jeunesse, afin de réformer la politique de l'enfance et de la jeunesse, notamment par un meilleur encadrement des dépenses et la mise en place de nouvelles règles de financement par les CAF. L'objectif est de mieux cibler les interventions de ces dernières en matière $d$ 'accueil en visant les publics et les territoires les plus démunis, en fonction de critères de sélectivité. Ces nouvelles règles limitent le taux de financement des CAF à $55 \%$ du reste à charge après déduction de la prestation de service unique, des subventions éventuelles et de la participation des familles, au lieu de $70 \%$ antérieurement. Cette baisse de financement conduit les municipalités à redéfinir leurs priorités, à optimiser leurs investissements, à revoir leurs sources de financements et à chercher de nouveaux partenaires. Progressivement, la commune - ou la ville - s'est imposée comme le niveau pertinent de pilotage de l'accueil, où se confrontent l'offre et la demande et où les partenariats se mettent en place. Les transformations de la gouvernance locale induites par ces évolutions peuvent cependant générer des tensions entre les logiques qui prévalent dans I'accueil des enfants, notamment entre une logique de "service public » portée par les 


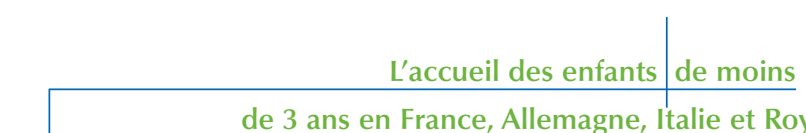

\begin{tabular}{|c|c|c|c|c|}
\hline & Allemagne & France & Italie & Royaume-Uni \\
\hline \multicolumn{5}{|l|}{ Enfants 0-2ans (révolus) } \\
\hline - Modes d'accueil formels (1) & & & & \\
\hline - 1 heure à 29 heures & 8,0 & 14,0 & 10,0 & 28,0 \\
\hline$->30$ heures & 8,0 & 17,0 & 16,0 & 5,0 \\
\hline \multicolumn{5}{|l|}{ - Autres arrangements (1) } \\
\hline - 1 heure à 29 heures & 24,0 & 15,0 & 22,0 & 31,0 \\
\hline - > 30 heures & 3,0 & 14,0 & 13,0 & 8,0 \\
\hline - par un-e assistant-e maternel-le (1) & 7,0 & 13,0 & 5,0 & 10,0 \\
\hline - par famille ou amis (1) & 22,0 & 20,0 & 32,0 & 31,0 \\
\hline $\begin{array}{l}\text { - gardés par les parents (seuls) } \\
\text { - Nombre moyen d'heures d'accueil (2) }\end{array}$ & 62,0 & 50,0 & 50,0 & 45,0 \\
\hline - accueil formel (en heures /semaine) & $n r$ & 28,4 & 30,9 & 14,4 \\
\hline - Autre accueil & $n r$ & 26,9 & 23,1 & 17,3 \\
\hline
\end{tabular}

Source: Eurostat, EU-SILC 2006 (European Communities, 2009)

Modes formels : tous les établissements d'accueil des jeunes enfants; autres arrangements : par une assistante maternelle ou un membre de la famille autre que les parents ; nr : non-réponses.

(1) En \% de tous les enfants âgés de moins de 3 ans.

(2) En nombre moyen d'heures par semaine.

institutions et une logique «marchande » d'adaptation à la demande (6) (Fenet, 2010).

Au Royaume-Uni, la loi de 2004 sur les enfants (Children's Act) stipule que c'est aux autorités locales que revient la charge de mettre en place le plan intégré pour l'enfance et la jeunesse (Children and Young People's Plan-CYPP). Ce plan stratégique place I'ensemble des services de l'enfance et de la jeunesse sous la responsabilité des collectivités locales, incluant la santé et la prévention de la criminalité. La loi de 2006 sur I'accueil des jeunes enfants (Childcare Act) attribue en outre aux municipalités la responsabilité d'assurer le bien-être des enfants, en veillant en particulier à ce que l'offre d'accueil soit suffisante pour répondre aux besoins. Les partenariats locaux qui se sont développés dans la foulée des réformes engagées depuis la fin des années 1990 impliquent surtout des acteurs publics, en lien avec I'objectif prioritaire de lutte contre la pauvreté des familles et des enfants. Ces acteurs sont des professionnels de l'accueil des enfants, des représentants des services de soins et d'assistance sociale, des services sociaux et d'autres institutions travaillant avec les enfants et les familles. Les entreprises en sont absentes, leur investissement étant lié à d'autres priorités et à d'autres outils. À la différence de l'Allemagne où les réformes de la politique familiale ont été menées avec l'appui du patronat et des syndicats, au Royaume-Uni, elles se sont réalisées avec I'appui des associations et des organisations non gouvernementales (ONG) présentes dans le champ de l'enfance et de la parentalité, porteuses de revendications en terme de reconnaissance du droit des enfants. L'action de ces «lobbies» a été d'asseoir la politique du New Labour sur le paradigme de l'investissement social (Williams et Roseneil, 2004).

Au total, en dépit de la diversité des priorités, les réformes des politiques d'accueil ont cherché à mobiliser de nouveaux acteurs, en particulier les entreprises, non seulement pour élargir leurs possibilités de financement mais aussi pour les responsabiliser autour d'enjeux de conciliation entre travail et vie familiale, d'enjeux démographiques et de cohésion sociale.

\section{L'accueil des enfants dans les quatre pays}

En dépit de la volonté affichée de développer l'offre d'accueil des enfants, des disparités persistent entre les quatre pays, non seulement au regard du nombre d'enfants accueillis mais aussi du temps d'accueil. Le tableau ci-dessus distingue l'accueil formel (crèche, halte-garderie, assistante maternelle, garde à domicile) et l'accueil informel (salarié non déclaré, au pair, membres de la famille ou de I'entourage) des enfants de moins de 3 ans. C'est en France et au Royaume-Uni que les recours aux modes d'accueil formels sont les plus courants, puisque $44 \%$ des enfants de cet âge y sont accueillis en France et $43 \%$ au Royaume-Uni contre $31 \%$ en Italie et $23 \%$ en Allemagne. En revanche, en Allemagne, la garde par les parents est la plus fréquente : elle concerne $62 \%$ des enfants de moins de 3 ans, contre $50 \%$ en France et en Italie et $45 \%$ au Royaume-Uni (à l'exclusion de tout autre mode d'accueil). En ce qui concerne les enfants de plus de 3 ans, plus de huit enfants sur dix sont accueillis dans une structure formelle, soit à plein temps (la majorité des enfants en Allemagne, en France, au

(6) À la différence de l'Allemagne, la France a opté pour le maintien des services d'accueil des enfants dans le champ de la Directive "services». Cette option place ces services dans une logique de marché intérieur pour le développement de l'offre. Les associations s'en trouvent affectées, passant d'une situation de partenaires des collectivités locales à une situation de prestataires par rapport à la commande publique, notamment par le biais des appels d'offre qui stimulent la concurrence pour l'obtention de marchés. 
Royaume-Uni) ou principalement à temps partiel (Italie). II importe toutefois de noter que ces chiffres ne coïncident pas exactement avec ceux que l'on peut collecter directement auprès de sources nationales. Celles-ci indiquent, par exemple, qu'en Allemagne, seulement $13,7 \%$ des enfants âgés de moins de 3 ans sont accueillis dans une structure collective (12\%) ou chez une assistante maternelle $(1,6 \%)$. Ces chiffres recouvrent néanmoins d'importantes disparités géographiques puisque ces modes d'accueil concernent $40 \%$ des enfants dans les Länder de l'Est contre $8 \%$ dans ceux de I'Ouest (Statistisches Bundesamt, 2006). En France, $67 \%$ des enfants de moins de 3 ans sont gardés principalement par les parents (63\%) ou un membre de la famille, tandis que $18 \%$ sont accueillis chez une assistante maternelle, $10 \%$ dans un établissement d'accueil des jeunes enfants, $2 \%$ vont à l'école maternelle et $2 \%$ sont gardés par une employée à domicile (Ananian et Robert-Bobée, 2009). En Italie, en 2005, 13,8\% des enfants de moins de 3 ans étaient accueillis dans une crèche, publique ou privée, (ISTAT, 2005).

Selon les pays, le statut des gestionnaires d'établissements d'accueil est soit public, soit privé. En Allemagne, plus de $63 \%$ des places d'accueil collectif destinées aux enfants de moins de 6 ans sont offertes par des prestataires privés dont les deux tiers environ sont à but lucratif (Statistisches Bundesamt, 2006). En France, 70 \% des structures monoaccueil sont gérées par des collectivités territoriales (62\% par des communes), $26 \%$ par des associations, $2 \%$ par des CAF et $1 \%$ par des entreprises privées commerciales. Quant aux structures multiaccueil, $56 \%$ sont gérées par des communes, $36 \%$ par des associations, $3 \%$ par une entreprise privée commerciale et $1 \%$ par une CAF (Bailleau, 2010). En Italie où les crèches privées se sont développées à un rythme accéléré au cours de la dernière décennie, cellesci représentent 38,9\% des structures en 2004, contre $6,7 \%$ en 1992 . On peut parler à ce propos $d^{\prime}$ un mouvement de privatisation des structures collectives d'accueil. Au Royaume-Uni, d'après le National Audit Office, le nombre de places d'accueil offert par le secteur privé pour les enfants d'âge préscolaire (0-4 ans) a augmenté de $90 \%$ entre 1997 et 2004, les trois quarts de ces places étant offertes par des entreprises privées ou le tiers secteur, et le reste par les collectivités locales et les écoles (Bryson et al., 2006). Au total, la confrontation de ces données montre l'emprise variable du privé, lucratif et non lucratif, sur le développement des modes d'accueil collectif. Enfin, si on considère plus précisément l'offre d'accueil des entreprises, les résultats de l'enquête de la Fondation européenne pour l'amélioration des conditions de vie et de travail de Dublin réalisée en 2004-2005 (Letablier et al., 2008) montrent que l'offre des entreprises en matière de crèches est la plus généreuse au Royaume-Uni, $6 \%$ des entreprises proposant un tel service à leurs salariés, contre moins de $2 \%$ dans les autres pays (graphique ci-dessous). En France, les crèches d'entreprises sont en nombre limité comme l'indique l'enquête Familles et employeurs de l'Institut national d'études démographiques, et restent localisées dans des secteurs d'activité bien définis comme les hôpitaux (Pailhé et Solaz, 2009). En revanche, les entreprises, que ce soit du fait direct de l'employeur par l'intermédiaire de la gestion du personnel ou de celui des comités d'entreprise, tendent à s'engager dans le soutien à l'accueil des enfants.

\section{L'appel aux entreprises : des modalités diverses}

Si la déclinaison des objectifs de Barcelone s'inscrit dans des contextes - et développe des outils - différenciés, l'appel aux entreprises pour qu'elles s'engagent dans le développement de l'offre d'accueil des jeunes enfants est un point commun des réformes. Outre la volonté affichée de diversifier les acteurs intervenant dans ce champ politique, il s'agit de faire appel à de nouveaux financeurs,

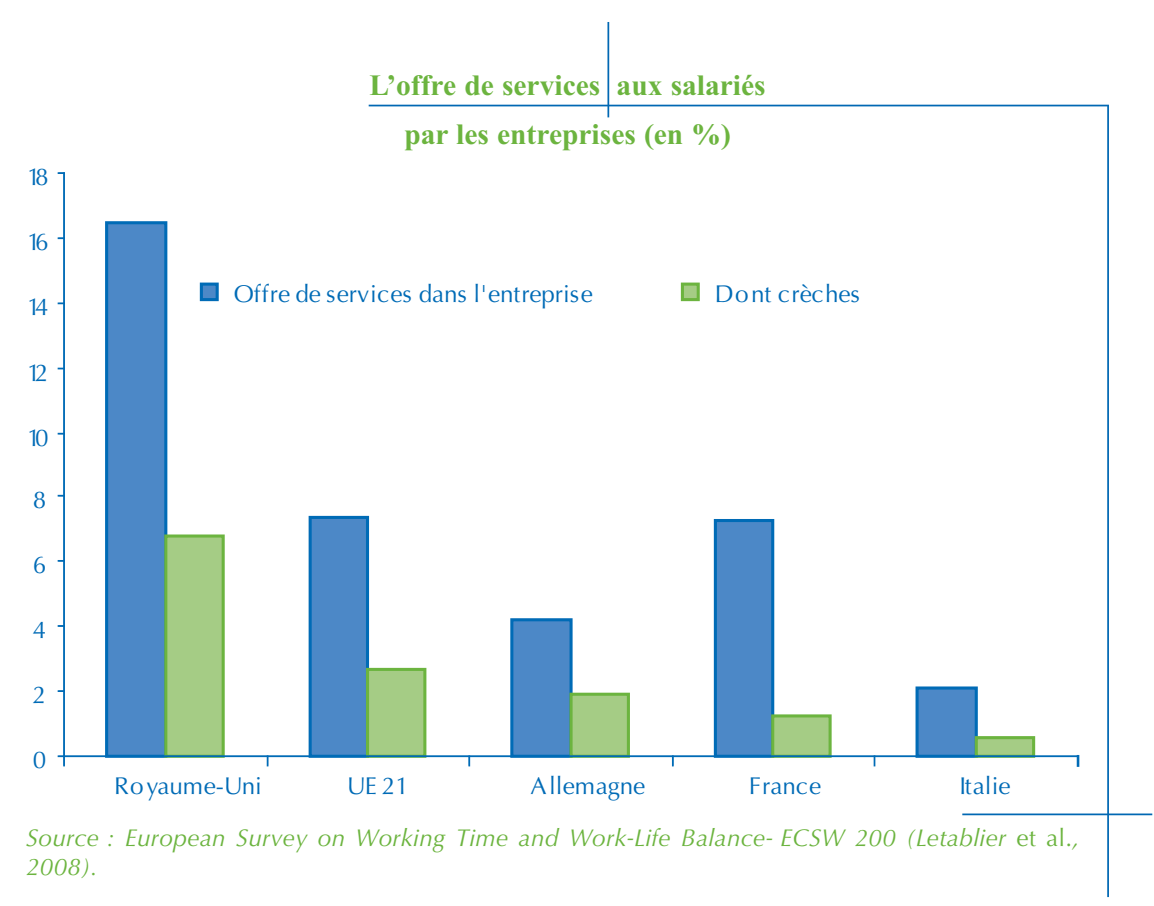


de promouvoir un "choix » important de modes $d$ 'accueil pour les parents, $d$ 'associer davantage les partenaires sociaux ainsi que la société civile dans ce qui est construit parfois (en Allemagne par exemple) comme une nouvelle " cause nationale».

\section{Différentes logiques d'implication}

Si l'appel aux entreprises pour qu'elles s'investissent davantage dans le développement des modes d'accueil des enfants, et au-delà dans le soutien à la parentalité de leurs salariés, est commun aux quatre pays, les modalités de leur implication répondent à des logiques différentes en fonction des contextes institutionnels et politiques et, surtout, en fonction des relations avec la puissance publique.

\section{Une logique de responsabilité partagée}

En Allemagne, les entreprises ont été directement mobilisées dans les réformes de la politique familiale, en amont dans le processus de décision comme en aval dans la mise en ouvre de la politique (Veil, 2010 ; Klammer et Letablier, 2008). Les partenaires sociaux sont parties prenantes de la nouvelle politique familiale au niveau de sa conceptualisation comme à celui de sa mise en œuvre. Deux réformes adoptées après le sommet de Barcelone traduisent un changement majeur dans la conception de la relation entre parentalité et emploi. II s'agit, d'une part, de l'instauration en 2007 d'un droit opposable à un service de garde pour les enfants de moins de 3 ans (ce droit existait depuis 1995 pour les 3-6 ans) adopté à l'issue d'un "sommet des crèches » réunissant les représentants de l'État, des Länder et des communes et, d'autre part, de la réforme du congé parental raccourci à douze mois mais rémunéré à hauteur de $67 \%$ du salaire antérieur, qui s'applique aux enfants nés depuis le $1^{\mathrm{er}}$ janvier 2007. Dans un contexte marqué par une faible fécondité et par une pénurie d'emplois qualifiés, ces réformes inscrivent le modèle de famille avec deux parents actifs sur l'agenda sociétal, en rupture avec la tradition d'activité professionnelle réduite, voire d'inactivité des mères allemandes. II s'agit désormais de permettre aux mères de rester en emploi et aux actives de ne pas renoncer à la maternité.

Ce changement de cap a pris la dimension d'une cause nationale, mobilisant un large panel d'acteurs : I'État, les partenaires sociaux, les associations caritatives et familiales, les fondations. Dès 1999, des "pactes locaux pour la famille » se sont constitués afin de faire évoluer les mentalités et les attitudes, tant des collectivités locales que des entreprises, vis-à-vis de la parentalité et l'accueil des enfants. L'«Alliance pour les familles» créée en 2003 à I'initiative du ministère de la Famille, et présidée par le président du patronat allemand, le BDI (Bundesverband der Deutschen Industrie e.V.) vise à promouvoir l'action des entreprises en faveur des familles. Le programme "Famille, facteur de succès » (Erfolgsfaktor Familie) lancé en 2006 par le ministère de la Famille, en coopération avec le patronat et les syndicats, comporte un volet spécifique pour les petites et moyennes entreprises : pendant deux ans, à partir de 2009, les entreprises se verront restituer $50 \%$ des coûts de fonctionnement des infrastructures de garde qu'elles offrent par un financement du fonds social européen. Pour mener à bien la « modernisation » de sa politique familiale et assumer ce tournant idéologique, le gouvernement allemand a sollicité les entreprises qui ont répondu avec le souci non seulement $d$ 'assurer leurs ressources en main-d'œuvre, mais aussi dans un esprit de responsabilité sociale, tant au niveau macro (national) qu'au niveau micro, celui de la gestion de leur ressources et de leur notoriété (Klenner, 2005). Enfin, tant I' «Alliance » que les «pactes locaux» pour les familles s'inscrivent dans une logique territoriale de développement local visant à réunir l'ensemble des acteurs concernés par la petite enfance pour mettre en commun leurs idées et se coordonner pour élaborer des projets, selon une approche territorialisée de I'action publique. Un rôle de coordination peut éventuellement être endossé par l'exécutif municipal qui attribue un rôle variable aux employeurs : ce rôle apparaît relativement marginal pour la ville de Francfort, mais central dans celle d'Eschborn où les employeurs cofinancent et coplanifient l'offre de services à la petite enfance (Veil et Rouault, 2009).

\section{Une logique de complémentarité-contractualisation} Cette logique, qui se traduit par I'instauration d'une forme contractualisée de relations publicprivé, est particulièrement mise en évidence en France où le gouvernement a cherché à étendre les partenariats entre les CAF et les collectivités locales à d'autres acteurs telles les associations et les entreprises. La régulation des relations passe par le contrat, afin d'instaurer une complémentarité des partenaires. On pourrait aussi parler de «logique partenariale ", dans un cadre fortement réglementé. De fait, la contractualisation avec les CAF est l'outil de la politique de l'enfance (et de la jeunesse), contribuant à structurer l'offre locale d'accueil et à construire une politique avec les partenaires locaux. Les projets sont formalisés autour d'objectifs et de priorités financières et politiques. Ils mobilisent des outils qui permettent de fédérer ces objectifs. Les employeurs contribuent également au financement de la politique familiale par I'intermédiaire des cotisations sociales et participent à la gestion des CAF dans le cadre du système paritaire de sécurité sociale. L'instauration d'un " crédit impôt familles » par la Loi de finances 2004 permet aux entreprises de s'impliquer davantage dans le développement des modes d'accueil en rendant cet investissement attractif puisqu'elles peuvent déduire de leurs bénéfices les dépenses 
dédiées à la création ou au fonctionnement de places d'accueil. Parallèlement, les fonds spéciaux d'aide à l'investissement dans l'accueil des jeunes enfants, jusque-là réservés aux collectivités locales et au secteur associatif, ont été ouverts aux entreprises à but lucratif que sont les entreprises de crèches. L'appel aux entreprises, en France, interpelle à la fois le service des ressources humaines et les comités d'entreprises, chacun ayant sa logique propre : une logique gestionnaire dans le premier cas et une logique sociale dans I'autre. Ce partage des responsabilités ne se retrouve pas dans les autres pays.

Les contrats enfance et jeunesse, qui ont remplacé en 2006 les contrats enfance et les contrats temps libres, sont l'instrument de cette logique contractuelle. Dans un contexte où la régulation tutélaire est traditionnellement forte, les principaux outils de coordination entre les divers acteurs sont des dispositifs descendants, institués au niveau central mais sous la responsabilité des autorités municipales et départementales. Au niveau municipal, ces contrats sont des outils de négociation bilatérale, ou tripartite, entre financeurs (CAF, mairies, associations et, plus récemment, entreprises). En outre, depuis 2002, au niveau départemental, des commissions départementales d'accueil des jeunes enfants (CODAJE) réunissent, sous la présidence du président du conseil général et la vice-présidence de la CAF, I'ensemble des acteurs concernés par la petite enfance: représentants du ministère de l'Éducation nationale, de la Jeunesse et des Sports, des communes, des associations gestionnaires, professionnelles ou familiales, des salariés et des employeurs, afin de favoriser la cohérence des politiques et des actions en matière de développement des modes d'accueil, de veiller à leur adaptation aux besoins des familles ainsi qu'à l'égalité d'accès et à la qualité des modes d'accueil. Toutefois, le fait que les acteurs non institutionnels - entreprises privées, parents ou encore professionnels - restent peu intégrés à ces structures de gouvernance explique en partie les disparités territoriales de développement des structures d'accueil (Fraisse et al., 2008).

\section{Une logique de substitution}

Dans les pays où l'État joue un rôle plus faible en matière de politique familiale, soit parce que ce n'est pas le niveau pertinent de régulation comme en Italie, soit parce que l'État laisse l'initiative à $d^{\prime}$ autres acteurs comme au Royaume-Uni, les entreprises jouent un rôle important. Mais ce rôle n'est que faiblement, voire pas du tout, coordonné avec celui des pouvoirs publics. Les entreprises agissent en fonction de leurs intérêts, mues surtout par une logique de gestion de leurs ressources humaines, cherchant à retirer de cet engagement un avantage concurrentiel en termes d'attractivité de la maind'œuvre.
En Italie, malgré la mise en place de programmes de financement à la fin des années 1990, et d'un "plan extraordinaire pour le développement de crèches » en 2006, I'accueil des jeunes enfants hors de la famille reste limité. Non seulement les taux de couverture sont faibles mais ils sont très variables selon les régions : en 2005, les crèches municipales accueillent environ $2 \%$ des enfants de moins de 3 ans dans les régions du sud, $12 \%-13 \%$ dans les régions du centre et du nord (ISTAT, 2008). L'investissement des entreprises, comme celui du secteur associatif, s'inscrit dans cette défaillance de l'acteur public. En 2005, les crèches privées représentent près de $40 \%$ de l'ensemble des structures collectives (ISTAT, 2005). Depuis le début des années 2000, outre des fonds d'investissement nationaux, votés chaque année dans la loi de finances, des incitations fiscales soutiennent les entreprises créant des places d'accueil. Parallèlement, depuis 2005, les parents salariés bénéficient de réductions (ou de crédits) d'impôts pour les inciter à faire garder leurs enfants. Mais ce sont surtout les grandes entreprises et en particulier les entreprises publiques (hôpitaux, universités, municipalités, provinces), situées dans les régions du nord, qui ont créé des places d'accueil, en ayant recours à un opérateur extérieur comme les entreprises de crèches ou les consortia de coopératives. Jusqu'à présent, en Italie, comme en Allemagne, la petite enfance $n^{\prime}$ est pas réellement constituée en champ politique, les compétences et les responsabilités s'inscrivent dans un principe constitutionnel de subsidiarité en cascade. Depuis le début des années 1990, I'offre privée a augmenté en réponse à l'accroissement de la demande et à la faiblesse de l'offre publique. Cette offre privée, associative ou lucrative, s'est d'abord développée de façon autonome et ce n'est que depuis le début des années 2000 qu'elle est soutenue par des fonds incitatifs. Mais, tant du point de vue de I'organisation territoriale de la programmation que des financements, la situation reste floue en raison notamment des objectifs contradictoires contenus dans les réformes des institutions. II en résulte de fortes disparités régionales en fonction de l'orientation politique et de la volonté d'agir des gouvernements régionaux (Pavolini, 2005). La diversité est plus grande encore au niveau des municipalités qui assument la responsabilité financière des services d'accueil (Trancart et al., 2006).

De fait, I'Italie s'inscrit dans une logique de délégation-substitution: les autorités publiques laisent se déployer les initiatives privées qu'elles commencent à soutenir financièrement. Le welfaremix qui en résulte assemble des initiatives locales venant de la société civile et des mouvements de sous-traitance de l'offre - publique, par délégation, ou privée, par appels d'offre - à des entreprises de crèches. Les différents acteurs, publics et privés, se confrontent au sein de partenariats complexes, 
chaque partenaire ayant des exigences différentes à chaque étape du projet : montage, investissement, fonctionnement.

\section{Une logique de juxtaposition}

Au Royaume-Uni, au tournant des années 1990-2000, les gouvernements travaillistes ont engagé des réformes qui constituent ce qu'ils ont eux-mêmes appelé une "révolution "-Childcare Revolution (Finding, 2010), faisant de la garde des enfants une affaire publique. Les plans successifs, en 1998 ( National Childcare Strategy ») puis en 2004 ("Ten years strategy »), ont amélioré l'accès aux congés, maternité et parental, et ont instauré l'accès à une préscolarisation à temps partiel, gratuite, pour les enfants âgés de 3 ans à 4 ans (l'école primaire commençant à l'âge de 5 ans). Les collectivités locales sont en effet tenues désormais d'offrir douze heures trente hebdomadaires (qui devraient passer à quinze heures en septembre 2010) de préscolarisation aux enfants de 3 ans révolus, pendant trente-huit semaines. Pour atteindre cet objectif, les collectivités locales peuvent acheter ces places aux prestataires de leur choix. Moins de $60 \%$ de ces places seraient offertes par le secteur public en Angleterre, et un peu plus en Écosse, au pays de Galles et en Irlande du Nord (Babyliss et Sly, 2009; Department for Education, 2010). Par ailleurs, cet accueil se fait massivement à temps partiel : I'enquête Childcare Survey of Parents de 2008 indique que les enfants de 0 à 4 ans accueillis dans des modes de garde formels le sont pour des durées de dix à quinze heures hebdomadaires; ces chiffres incluant la préscolarisation gratuite des 3-4 ans, qui est le mode de garde le plus utilisé, laissent supposer des durées sensiblement plus courtes pour les 0-2 ans. Selon Chris Pascal et Tony Bertram (2010, p. 83), la loi de 2006 définit ainsi « un nouveau rôle des autorités locales, celui de réguler l'offre d'accueil tout en ne leur permettant pas de fournir elles-mêmes ces services... "(Childcare Act 2006). Les autorités locales sont chargées de veiller au bon fonctionnement du marché et à la satisfaction des besoins. Il n'existe donc pas de collaboration directe avec les entreprises. Celles qui s'engagent dans cet investissement le font dans une perspective de gestion de leurs ressources humaines (sur les logiques d'engagement des entreprises, voir Ollier-Malaterre, 2008).

Ces quatre configurations de relations État-entreprises soulignent des modes de recomposition des relations public-privé différenciés selon les pays. Un jeu d'oppositions se dessine entre une logique de contractualisation-complémentarité public-privé, d'une part, et une logique de juxtaposition/substitution qui passe par une délégation des responsabilités, d'autre part. Ces logiques sont servies par différents instruments : contrats de partenariat, chèques services...
Vers un marché des services d'accueil?

Les pouvoirs publics soutiennent l'implication des entreprises dans le développement des structures d'accueil des jeunes enfants par des incitations financières et fiscales et des allègements de cotisations sociales visant à réduire sensiblement le coût de leur investissement. Parallèlement, les entreprises contribuent à solvabiliser la demande de leurs salariés parents, notamment au moyen de chèques services prépayés que ceux-ci peuvent utiliser pour acheter des services de garde d'enfants sur le marché. Cette forme d'implication des employeurs, plus ou moins diffusée selon les pays, est congruente avec la logique politique affichée d'accroître le choix des parents, à l'opposé de la logique "service public » de la petite enfance qui prévaut dans les pays nordiques.

\section{L'implication dans la solvabilisation des salariés- parents : le succès croissant des chèques services} Les " vouchers » et chèques services prépayés sont des instruments privilégiés par les entreprises et les comités d'entreprises, ou leur équivalent, pour s'impliquer dans l'accueil des enfants. Ce système s'est particulièrement développé au Royaume-Uni et en France ; il accompagne le processus de diversification des modes d'accueil, en lien avec la promotion du choix des parents. II apparaît comme le vecteur d'une logique marchande dans ce secteur, tout en relevant des politiques sociales d'entreprises. La réforme des chèques services en France a fortement contribué à diffuser cet outil de paiement des services d'accueil. Créé par la loi «Borloo» de 2005 pour favoriser le développement des services à la personne, le chèque emploi service universel (CESU) est largement utilisé par les entreprises sous sa forme préfinancée comme moyen de contribuer au paiement de services, notamment de services d'accueil, de leurs salariés parents. Ce titre de paiement à montant prédéfini, financé tout ou partie par un employeur (public ou privé), un comité d'entreprise, une mutuelle, une caisse de retraite ou une collectivité territoriale, permet ainsi d'alléger le coût des services pour les familles. De son côté, l'employeur bénéficie d'avantages fiscaux sur les aides versées à ses salariés par CESU. Ces chèques services sont particulièrement prisés par les comités d'entreprise. Ce système est également bien développé au RoyaumeUni sous la forme de "vouchers » dont le principe est le même qu'en France. Introduit en 2006, il permet aux salariés d'utiliser des chèques prépayés par leur employeur pour régler une structure d'accueil, publique ou privée, individuelle ou collective, à condition que la structure soit agréée. Ces chèques comportent des avantages fiscaux pour I'employeur comme pour les salariés. Ce système est partie prenante de la « révolution » des politiques publiques britanniques en direction de la petite enfance, incitant les entreprises à s'impliquer dans 
la mise en place d'un marché de l'accueil (DauneRichard et Letablier, 2009). L'utilisation de ces "vouchers » reste cependant plus répandue dans les grandes entreprises : d'après John Faragher (2008 a et b), $88 \%$ des entreprises de plus de mille cinq cents salariés en offrent contre $49 \%$ de celles employant moins de cinquante salariés.

Le souci des politiques d'accueil de répondre aux besoins toujours plus diversifiés des parents, ainsi que leur engagement à promouvoir un choix de plus en plus large de modes d'accueil, conduit à la création d'un marché des services d'accueil de la petite enfance où les demandeurs-parents doivent avoir la possibilité de choisir leur prestataire avec l'appui des aides publiques, tandis que les offreurs (à but lucratif ou non) se trouvent mis en concurrence, notamment par les procédures d'appel d'offres. Dans les quatre pays, l'offre privée se développe, soutenue à la fois par des incitations fiscales et par des crédits incitatifs nationaux, régionaux ou municipaux. Cette offre privée est le fait du secteur associatif et, de plus en plus, des entreprises de crèches qui ont émergé au cours des dernières années et qui tendent désormais à se regrouper en chaînes nationales et/ou internationales. Elles offrent leurs services aux entreprises et aux collectivités locales pour créer des structures d'accueil et/ou les gérer. Les entreprises, comme les municipalités, délèguent de plus en plus la création ou la gestion de structures d'accueil à ces entreprises de crèches plutôt que d'en assurer elles-mêmes la responsabilité (DauneRichard et al., 2007). Cette délégation contribue à I'instauration d'un marché des services d'accueil mettant en concurrence les associations (non lucratives) avec des entreprises à but lucratif, par le truchement des procédures d'appel d'offres. La pénétration du marché de l'accueil par ces entreprises de crèches s'accompagne de la diffusion de pratiques gestionnaires fondées notamment sur une rationalisation du pilotage et l'optimisation du remplissage. Cette ouverture aux prestataires privés à but lucratif a été particulièrement effective en Allemagne et en Italie où elle accompagne le développement des structures d'accueil. Elle est en progression en France et au Royaume-Uni. Dans les quatre pays, cette ouverture génère un glissement vers des formes de gouvernance plus marchandes et plus concurrentielles.

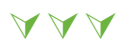

\section{Conclusion}

Le développement de I'offre d'accueil des enfants a été au cours de la dernière décennie une préoccupation commune aux pays de l'Union européenne et en particulier aux pays qui, comme I'Allemagne, le Royaume-Uni ou I'Italie, n'avaient pas de politique explicite et instituée d'accueil des enfants, ou comme la France où l'offre d'accueil reste insuffisante. Les réponses apportées à l'augmentation des besoins varient selon les pays non seulement en fonction des objectifs et des priorités assignées aux politiques mises en place mais aussi en fonction des outils mobilisés pour atteindre ces objectifs, et des acteurs impliqués. L'instauration d'une politique d'accueil des enfants a été un changement profond dans la conceptualisation du rôle de l'État dans ce domaine, comme dans l'approche de l'enfance et dans les représentations de leur éducation et de leur bien-être, en particulier en Allemagne (ex-Allemagne de I'Ouest) et au Royaume-Uni où cette question était essentiellement l'apanage de la famille. Dans ces deux pays, la mise en place d'une politique d'accueil des enfants supposait un changement radical des mentalités, notamment au regard de l'éducation des enfants et du rôle des mères. En France, où existait une politique d'accueil, les réformes ont été moins radicales et ont surtout porté sur les instruments de gouvernance et de pilotage, notamment sur les conditions de financement.

Les quatre pays étudiés ici ont cependant en commun de caler leurs réformes sur un principe identique, celui de respecter et de mettre en œuvre la liberté de choix des parents, ce qui les distingue du modèle nordique fondé sur le développement $d^{\prime}$ un service public d'accueil articulé à des prestations essentiellement en nature. Cet objectif politique de libre choix s'accorde avec une stratégie de diversification des formes d'accueil, des partenaires et des formes de soutien aux parents.

L'appel aux entreprises pour qu'elles s'impliquent dans cet objectif de développement des modes $d^{\prime}$ accueil pour les enfants afin de permettre aux mères de participer en plus grand nombre au marché du travail est également un point commun aux quatre pays. Toutefois, la confrontation des logiques d'appel aux entreprises, comme de leur implication, montre que cette mobilisation s'effectue de manière différenciée selon les contextes institutionnels, politiques et sociaux. Alors qu'en Allemagne, les partenaires sociaux, le patronat et les syndicats, ont été associés très directement, au niveau fédéral comme au niveau local, à la " modernisation» des politiques familiales, faisant des entreprises un partenaire actif des réformes, tel n'a pas été le cas dans les autres pays, en particulier au Royaume-Uni où les relations Étatentreprises ont une teneur différente. Pour autant, I'action des entreprises dans ce domaine, comme dans le soutien à la parentalité en général, est désormais articulée à des objectifs politiques explicites à cet égard, contribuant ainsi à renforcer la légitimité managériale de leur action. En France, les entreprises s'insèrent dans l'action 
publique en tant qu'acteurs complémentaires, susceptibles de contribuer au financement et au développement des structures d'accueil, comme aussi en Italie bien que sous une forme moins encadrée.

Si les entreprises se sont saisies diversement de ces opportunités, leur implication se traduit par deux changements majeurs: l'émergence d'opérateurs privés à but lucratif dans le secteur de l'accueil, d'une part, et le développement d'outils tels que les chèques services qui contribuent à solvabiliser les salariés parents, d'autre part. Ainsi, si très peu $d^{\prime}$ entreprises offrent des structures d'accueil pour les enfants de leurs salariés sur leur site, un nombre croissant contribuent au financement de structures et à la réduction des coûts d'accueil pour les parents salariés. La diffusion de ces nouveaux outils et l'introduction de nouveaux acteurs font que l'on s'éloigne d'un système universel fondé sur les droits des enfants, avec le risque de le lier davantage au travail des parents et aux besoins diversifiés des entreprises. En outre, la pénétration des entreprises de crèches est porteuse d'une logique nouvelle, plus gestionnaire et plus marchande que la logique de l'intérêt général portée en particulier par les associations, ou a fortiori un service public.

En tout état de cause, le développement d'un marché des services d'accueil de l'enfance, marqué par des exigences de rationalisation, incite à repenser les attentes en terme de qualité de ces services, ancrées jusque-là sur des référents relationnels, voire domestiques.

\section{\begin{tabular}{|l|l}
\hline & \\
\hline Références & bibliographiques
\end{tabular}}

- Ananian S. et Robert-Bobée I., 2009, "Modes d'accueil et de garde des enfants de moins de 6 ans en France en 2007 ", Etudes et Résultats, n 678.

- Babyliss J. et Sly F., 2009, "Children and young people around the UK», Regional Trends, $\mathrm{n}^{\circ} 41$.

- Bailleau G., 2010, «L'offre d'accueil des enfants de moins de 6 ans en 2008 », Études et Résultats, n 715.

- Bennett J., 2010, Nouvelles perspectives des études internationales sur la petite enfance, Revue internationale d'éducation, $\mathrm{n}^{\circ}$ 53:31-41.

- Bryson C., Kazimirski A. et Southwood H., 2006, "Childcare and Early Years Provision: a study of parents' use, views and experiences », Research Brief RB723, London: DfES [Department for Education and Skills].

- Daune-Richard A.-M, Odena S. et Petrella F., 2007, « Entreprises et modes d'accueil de la petite enfance : innovation et diversification », CNAF, Dossier d'études, $n^{\circ} 91$.

- Daune-Richard A.-M. et Letablier M.-T. (dir.), 2009, "Concilier travail et famille : I'engagement des entreprises dans la prise en charge des enfants (Allemagne, France, Italie et Royaume-Uni) », CNAF, Dossier d'études, $\mathrm{n}^{\circ} 120$.

- Department for Education, 2010, "Provision for children under five years of age in England; January 2010 », Statistical First Release, Royaume-Uni.

- Esping-Andersen G. (avec B. Palier), 2008, Trois leçons sur l'État-providence, Paris, Seuil.

- European Communities, 2009, "The provision of childcare services. A comparative review of 30 European Countries » (http://ec.europa.eu/social).

- Eurostat, 2008, Child poverty and well-being in the EU. Current status and way forward, Office for Official Publications of the European Communities, Luxembourg.

- Fagan C. et Teasdale N., 2008, "The provision of child care services in the UK », External report commissioned by and presented to the EU Directorate-General Employment and Social Affairs, Unit G1'Equality between Women and Men', EWERC, The University of Manchester, UK.

- Fagnani J., 2010, Les réformes de la politique familiale en Allemagne: I'enjeu démographique, in Radioscopies de l'Allemagne 2010 (sous la dir. de Clouet L. M. et Stark H.), Paris, éditions de I'Institut français des relations internationales : 249-266.

- Fagnani J., Math A. et Meilland C., 2008, "Comparaison européenne des aides aux familles », Institut de recherches économiques et sociales, rapport de recherche pour la CNAF.

- Fagnani J. et Math A., 2007, Les réformes de la politique familiale en Allemagne. De nouveaux horizons pour les femmes?, Droit Social, n 5:630-36.

- Faragher J., 2008 a, "Childcare voucher schemes: Friend of the family », site Internet de Personnel Today : http://www.personneltoday.com

- Faragher J., 2008 b, « Lack of employee awareness hampers take-up of childcare voucher schemes », site Internet de Personnel Today : http://www.personneltoday.com

- Fenet F., 2010, Évaluer une politique locale d'accueil des jeunes enfants. L'expérience de la Ville de Nantes, Informations sociales, $n^{\circ}$ 160:84-91. 
- Finding S., 2010, La mise en place d'une politique de la petite enfance, Informations sociales, n 159:90-100.

- Fraisse L., Lhuillier V. et Petrella F., 2008, L'accueil des jeunes enfants en Europe : vers des formes de gouvernance multilatérale et intégrée ?, Revue française de socio-économie, $n^{\circ}$ 2:141-160.

- Giddens A., 1998, The third way: The renewal of social democracy, Cambridge: Polity Press.

- ISTAT, 2008, "L'indagine censuaria sugli interventi e i servizi sociali dei Comuni. Anno 2005 », Rome, (http://www.istat.it).

- ISTAT, 2005, « Rapporto annuale. La situazione del paese nel 2004 », Rome (http://www.istat.it)

- Klammer U. et Letablier M.-T., 2008, L'implication des entreprises et des partenaires sociaux dans l'aide aux familles pour concilier travail et vie familiale en Allemagne et en France, Recherches et Prévisions, $\mathrm{n}^{\circ}$ 92:73-85.

- Klenner C., 2005, Gleichstellung von Frauen und Männern und Vereinbarkeit von Familie und Beruf. Eine Analyse von tariflichen Regelungen in ausgewäh/ten Tarifbereichen, in WSI in der Hans Böeckler Stiftung (Hrsg.) WSI-Tarifhandbuch 2005, Frankfurt am Main, Bund-Verlag:39-65.

- Letablier M.-T., Lucy A., Math A. et Thévenon O., 2009, " The costs of children and the effectiveness of policies supporting parenthood in the European Union », rapport pour la Commission européenne (DG V), Paris, INED, Document de travail, $\mathrm{n}^{\circ} 158$.

- Letablier M.-T., Perraudin C., Anxo D., Fagan C. et Smith M., 2008, La prise en compte de la vie familiale de salariés par les entreprises. Une comparaison européenne, Recherches et Prévisions, $n^{\circ}$ 92:91-101.

- Lewis J., Tanner E. et Welsh E., 2006, The quality - defining process in early years' services. A case study, Children and Society, vol. 20, $\mathrm{n}^{\circ}$ 1:4-16.

- Odena S., 2009, «Les professions et leur coordination dans les établissements d'accueil collectifs du jeune enfant: une hétérogénéité source de tensions au sein des équipes », Cnaf, Dossier d'études, $\mathrm{n}^{\circ} 121$.

- OCDE, 2006, Starting Strong II. Early childhood education and care, Éditions de l'OCDE.

- OCDE, 2004, "Early childhood education and care policy in the Federal Republic of Germany ", note par pays de I'OCDE (http://www.oecd.org/dataoecd).

- Ollier-Malaterre A., 2008, Comment les employeurs appréhendent-ils la vie hors travail de leurs salariés? Une comparaison entre le modèle français et le modèle nord-américain, Recherches et Prévisions, n 92:47-60.

- Pailhé A. et Solaz A. (dir.), 2009, Entre famille et travail : des arrangements de couple aux pratiques des employeurs, Paris INED/La Découverte.

- Palier B., 2005, Vers un État d'investissement social. Pistes pour une redéfinition de la protection sociale, Informations sociales, $n^{\circ} 128: 118-128$.

- Pascal C. et Bertram T., 2010, Des services intégrés de l'enfance pour tous en Angleterre, Revue internationale d'éducation, $\mathrm{n}^{\circ}$ 53:77-87.

- Pavolini E., 2005, Stili regionali delle politiche in favore degli anziani, La Rivista delle Politiche Sociali, vol. 2, $\mathrm{n}^{\circ}$ 2:203-216.

- Statistisches Bundesamt, 2006, "Statistiken der Kinder-und Jugendhilfe ».

- Trancart D., Sabatinelli S., Fraisse L. et Boggi O., 2006, "Les déterminants du recours aux modes de garde : une comparaison France-Italie », rapport pour la Mission Recherche de la DREES.

- Unicef, 2008, "La transition en cours dans la garde et l'éducation des enfants. Tableau de classement des services de garde et d'éducation des jeunes enfants dans les pays économiquement avancés », Bilan Innocenti $n^{\circ} 8$, Centre de recherches Innocenti, Florence.

- Unicef, 2006, "La pauvreté des enfants dans les pays riches ", Innocenti Research Centre, Bilan n 7 , Centre de recherches Innocenti, Florence.

- Unicef, 2000, "Tableau de classement de la pauvreté des enfants parmi les nations riches", Innocenti Research Centre, Bilan n 1 , Centre de recherches Innocenti, Florence.

- Veil M., 2010, Allemagne. Le rôle accru des entreprises dans la nouvelle politique familiale, Chronique internationale de I'IRES, $\mathrm{n}^{\circ}$ 122:25-36

- Veil M. et Rouault S., 2009, Das Engagement der Betriebe in der Kleinkindbetreuung im DeutschFranzösischen Vergleich. http://www.boeckler.de/pdf_fof/S-2008-89-4-2.pdf

- Voisin J., 2009, Développement de la garde des enfants, IGAS, rapport n RM2009-033P, Paris, La Documentation française.

- Whiteford P. et Adema W., 2007, "What works best in reducing child poverty: a benefit or work strategy? », OCDE Social, Employment and Migration, Working Paper n 52.

- Williams F. et Roseneil S., 2004, Public values of parenting and partnering: Voluntary organizations and welfare policies in new labour's Britain, Social Politics, vol. 11, $\mathrm{n}^{\circ}$ 2:181-216. 
